ISSN 0103-5150

Fisioter. Mov., Curitiba, v. 24, n. 1, p. 47-56, jan./mar. 2011 Licenciado sob uma Licença Creative Commons

\title{
Avaliação da satisfação dos pacientes submetidos à intervenção fisioterapêutica no município de Campo Maior, PI
}

\author{
Satisfaction evaluation of patients underwent physical therapy \\ intervention in the city of Campo Maior, PI
}

\author{
Juscelino Rodrigues Gonçalves ${ }^{[a]}$, Felipe Eduardo Lages Veras ${ }^{[b]}$, \\ Adriana Cavalcanti de Macêdo Matos $^{[c]}$, Isnammya Silvinne Araújo Lima ${ }^{[\mathrm{d}]}$ \\ [a] Graduando do curso de Fisioterapia da NOVAFAPI, Teresina, PI - Brasil, e-mail: juscenina@yahoo.com.br \\ [b] Graduando do curso de Fisioterapia da NOVAFAPI, Campo Maior, PI - Brasil, e-mail: felipeduardoveras@hotmail.com \\ [c] Fisioterapeuta, docente do curso de Fisioterapia da NOVAFAPI, Teresina, PI - Brasil, e-mail: fisioadrica@yahoo.com \\ [d] Graduanda do curso de Fisioterapia da NOVAFAPI, Teresina, PI - Brasil, e-mail: isnammya@hotmail.com
}

\section{Resumo}

Introdução: Buscar satisfazer as necessidades dos usuários de serviços de saúde consiste em uma forma de medir a qualidade da atenção oferecida pelos profissionais desse setor, ainda que a qualidade da assistência continue sendo uma grande preocupação na saúde. Objetivo: Avaliar a satisfação dos pacientes em tratamento fisioterapêutico em quatro clínicas do município de Campo Maior, Piauí. Materiais e métodos: Foi realizado um estudo descritivo e transversal em uma amostra de 80 pacientes. A pesquisa consistiu na aplicação de um questionário contendo 20 perguntas objetivas para avaliação da satisfação dos serviços oferecidos pelas clínicas selecionadas. Resultados: Dos 80 entrevistados, 55\% eram do gênero feminino, 26,25\% pertenciam à faixa etária de 40 a 51 anos (26,25\%), 46,75\% fizeram o ensino fundamental, 66,75\% possuíam renda mensal de 1 a 3 salários minimos e 66,25\% não disponibilizavam de planos de saúde. A maioria dos indivíduos foi encaminhada por médicos e considerou a clínica de fácil acesso; 55\% utilizava transporte particular ou alugado para locomoção; e a indicação de terceiros foi o principal motivo de escolha do serviço (65\%). Os pacientes, em grande parte, mostraram-se satisfeitos com o tempo de espera para consulta, atendimento e uso dos aparelhos e confirmaram haver ótima relação com o fisioterapeuta. Os entrevistados ainda relataram ótima limpeza, conforto e boas instalações e consideraram suas necessidades de tratamento atendidas. Conclusão: No geral, observou-se grande satisfação dos usuários dos serviços de 
fisioterapia do município de Campo Maior. Entretanto, outros estudos se fazem necessários, correlacionando o grau de satisfação aos dados socioeconômicos.

Palavras-chave: Avaliação. Satisfação dos usuários. Serviços de saúde.

\section{Abstract}

Introduction: Search meet the needs of users of health services is as a way of measuring the quality of care offered by professionals in this sector, although the quality of care remains a major concern in health. Objective: To evaluate patient satisfaction in physical therapy at four clinics in the city of Campo Maior, Piauí. Materials and methods: We conducted a cross-sectional descriptive study and a sample of 80 patients. The survey consisted of a questionnaire containing 20 objective questions to assess satisfaction with services offered by clinics selected. Results: Of the 80 respondents about the socio-demographic profile was mostly female, age 40-51 years, had the level of elementary school education, monthly income 1-3 times the minimum wage, and most did not provide plans health. In the item accessibility was observed that most individuals were referred by physicians, as they judged as easy access to the serum, using mostly private transport or hire, did not show difficulties to attend, and details of others was the main reason for choosing the service. On the professional/ patient relationship, most respondents were satisfied with the waiting time for consultation, assistance and use of equipment, the service was very clear to patients, and they have confirmed there is a great relationship with the physiotherapist. Considering the ambience of the clinics, was judged as excellent cleanliness, comfort and buildings, users also reported having privacy during the visits and their treatment needs met. Conclusion: In general, there was great satisfaction of users of physiotherapy services of Campo Maior. However, other studies are necessary correlating the degree of satisfaction to the socio-economic data.

Keywords: Evaluation. Consumer preference. Health services.

\section{Introdução}

O Brasil vem enfrentando uma série de problemas no setor público de saúde, resultando em ineficiência dos serviços prestados à população que o utiliza, levando ao aumento de custos sociais e se fazendo necessário um monitoramento desses serviços para o processo de evolução do sistema de saúde brasileiro (1). Associada a isso, vem crescendo a importância do reconhecimento técnico sobre a perspectiva do usuário quando se aborda a qualidade de serviços de saúde (2).

Buscar satisfazer as necessidades dos usuários de serviços de saúde consiste em uma forma de medir a qualidade da atenção oferecida pelos profissionais desse setor, ainda que a qualidade da assistência continue sendo uma grande preocupação na saúde. Segundo Linder-Pel, a satisfação é a expressão de uma atitude, ou seja, uma resposta afetiva que está relacionada com a crença de que o serviço deve possuir componente e dimensão adequados, sendo, dessa forma, a satisfação definida como avaliação positiva do indivíduo acerca de dimensões distintas do serviço de saúde. Ultimamente, com a publicação de novos estudos, observa-se um crescente interesse nessa temática, porém, o conhecimento dos indicadores da satisfação do paciente com a assistência na rede pública em comparação com a privada ainda apresenta a necessidade de trabalhos aprofundados (3).

Mendonça e Guerra (4) realizaram um estudo com o intuito de traduzir para o português, adaptar culturalmente para a sociedade brasileira e legitimar o instrumento de medida de satisfação dos pacientes com a assistência fisioterapêutica recebida proposto por Goldstein et al. (5). A versão brasileira do instrumento apresentou confiabilidade e validez na avaliação da satisfação dos usuários dos serviços de fisioterapia das redes privada e pública de saúde. Posteriormente, os mesmos autores desenvolveram e legitimaram um instrumento de medida da satisfação do paciente usuário do serviço de fisioterapia ambulatorial específico para o contexto cultural brasileiro, contudo, ainda restrito à rede privada. 0 estudo forneceu uma ferramenta capaz de contribuir não somente em termos de gestão, mas 
também para o processo de mudança e planejamento, elementos esses necessários para aperfeiçoar a realidade de serviços da fisioterapia.

A implantação do Sistema Único de Saúde (SUS), em 1988, estabeleceu a necessidade de se repensar sobre o funcionamento desses serviços e de buscar a melhoria da qualidade - na intenção de atender às expectativas daqueles que utilizam os serviços -, buscando efetividade, eficiência, equidade, aceitabilidade, acessibilidade e adequabilidade (6).

Por se tratar da satisfação do usuário, um componente que intervém na qualidade de vida dos indivíduos, propõe-se neste estudo caracterizar tal resposta por meio de uma avaliação de satisfação nos sujeitos submetidos a algum tratamento fisioterapêutico.

\section{Materiais e métodos}

Trata-se de uma pesquisa do tipo descritiva e transversal sobre avaliação da satisfação dos pacientes submetidos à intervenção fisioterapêutica no município de Campo Maior, Piauí. Tal município é polo de saúde para as cidades vizinhas, pois as pessoas procuram algum tipo de tratamento de saúde, inclusive o fisioterapêutico, por não ter profissionais disponíveis em suas cidades de origem. A pesquisa foi realizada em quatro clínicas, no período de abril a maio de 2010, todas cadastradas junto ao Conselho Regional de Fisioterapia e Terapia Ocupacional da 6 ${ }^{\text {a }}$ Região, identificadas respectivamente com número de pacientes atendidos durante o mês: Clínica Cemed Fisio (40 pacientes, rede privada); Centro de Saúde Sandu (100 pacientes, rede municipal); Hospital Regional de Campo Maior (80 pacientes, rede estadual); e Clínica Nossa Senhora das Mercês (100 pacientes, filantrópica). A população de referência para o estudo é da cidade de Campo Maior, com 42.000 habitantes, e a população de estudo são pacientes em tratamento fisioterapêutico no município, com amostra randomizada aleatória de 20 pacientes em cada clínica, totalizando o número de 80 pacientes, mediante assinatura do documentoautorização do(a) diretor(a) da clínica e ou hospital.

Os critérios de inclusão utilizados na pesquisa foram: usuários de ambos os sexos, com idade superior a 18 anos, que estivessem submetidos a algum tipo de tratamento fisioterapêutico de no mínimo seis atendimentos. Foram excluídos aqueles com dificuldade de expressão (problemas de fala) ou de compreensão, e/ou que se recusassem a assinar o Termo de Consentimento Livre Esclarecido (TCLE).

A pesquisa foi realizada em três fases: exploratória, aplicação do questionário e análise dos resultados, cada fase agregando diferentes ciclos de pesquisa. A primeira fase consistiu na coleta de informações científicas em bancos de dados nos portais BIREME, SciELO, Biblioteca Cochrane, MEDLINE e LILACS e em bibliografias do inventário da biblioteca da Faculdade de Saúde, Ciências Humanas e Tecnológicas do Piauí (NOVAFAPI).

A segunda fase consistiu na aplicação de um questionário, elaborado por Machado (7), o qual foi modificado para realização desta pesquisa e foi direcionado aos pacientes durante o atendimento a fim de coletar os dados necessários, sem comprometimento do funcionamento dos serviços. Esse instrumento de coleta, contendo questões fechadas, está estruturado em quatro partes: perfil sociodemográfico, acessibilidade, satisfação do usuário e ambiência, com cinco perguntas cada uma, totalizando vinte perguntas diretas e objetivas, preservando, de acordo com a resolução 196/96, as identidades dos pacientes, assim como a fidelidade dos dados recolhidos, ressaltando que o emprego desses dados será somente para fins previstos nesta pesquisa.

$\mathrm{Na}$ terceira fase foram calculadas frequências para as variáveis categóricas, recorrendo-se ao software Office Excel (versão ano 2005) para as análises estatísticas descritivas e médias para variáveis contínuas, como idade dos pacientes, tempo para ser admitido na consulta e tempo para ingressar na intervenção.

A pesquisa procedeu de acordo com as diretrizes e normas regulamentadas na resolução 196/96 do Conselho Nacional de Saúde e iniciou após a apreciação do Comitê de Ética em Pesquisa (CEP) da Faculdade NOVAFAPI, recebendo parecer de aprovado de acordo com o protocolo CAAE n. 0195.0.043.000-09.

\section{Resultados e discussão}

A pesquisa foi realizada no período de abril a maio de 2010, em quatro clínicas de fisioterapia do município de Campo Maior, PI, por meio de um questionário aplicado, sendo tais clínicas de caráter distinto: particular, filantrópica, estadual e municipal. Ao todo foram entrevistados 80 pacientes, 20 em cada clínica. 
A população, de um modo geral, apresentou predominância do gênero feminino (55\%) e, em relação à idade, preponderaram indivíduos com 40 anos ou mais. Quanto ao nível de escolaridade, a maioria dos entrevistados $(43,75 \%)$ tem apenas o ensino fundamental, ressaltando-se que não houve discrepâncias entre os níveis de escolaridade em termos de quantidade entre os diferentes caracteres das clínicas (particular, filantrópica, estadual e municipal). No que se refere à renda familiar, $63,7 \%$ recebe de um a três salários mínimos, enfatizando-se que, da população atendida na clínica filantrópica, 50\% recebe de quatro a seis salários mínimos. Quanto ao convênio de saúde, apenas 33\% tem plano de saúde e a maioria dos entrevistados (66) não tem, embora desfrute do SUS (Quadro 1).

Em nosso estudo, não encontramos diferenças significativas no que condiz à renda familiar, no entanto, ao analisarmos a posse de convênios, observamos algo inesperado, dos pacientes atendidos em clínicas particulares poucos possuíam plano de saúde, ao contrário dos pacientes atendidos pela instituição filantrópica. Outro dado importante é que os indivíduos que usufruíam dos serviços filantrópicos, no geral, possuíam melhor escolaridade (Quadro 1).

Quadro 1 - Perfil sociodemográfico dos pacientes entrevistados em quatro clínicas no município de Campo Maior, PI (2010)

\begin{tabular}{|c|c|c|c|c|c|c|c|c|c|c|}
\hline \multirow[b]{2}{*}{ Dados avaliados } & \multicolumn{2}{|c|}{ Particular } & \multicolumn{2}{|c|}{ Filantrópica } & \multicolumn{2}{|c|}{ Estadual } & \multicolumn{2}{|c|}{ Municipal } & \multicolumn{2}{|c|}{ Total } \\
\hline & $n=20$ & $\%$ & $\mathrm{n}=\mathbf{2 0}$ & $\%$ & $n=20$ & $\%$ & $n=20$ & $\%$ & $n=80$ & $\%$ \\
\hline \multicolumn{11}{|l|}{ Gênero } \\
\hline Masculino & 9 & 45 & 10 & 50 & 10 & 50 & 7 & 35 & 36 & 45 \\
\hline Feminino & 11 & 55 & 10 & 50 & 10 & 50 & 13 & 65 & 44 & 55 \\
\hline \multicolumn{11}{|l|}{ Faixa etária } \\
\hline $18-28$ & 1 & 5 & - & - & 2 & 10 & 2 & 10 & 5 & 6,25 \\
\hline $29-39$ & 4 & 20 & - & - & 3 & 15 & 1 & 5 & 8 & 10 \\
\hline $40-51$ & 5 & 25 & 6 & 30 & 7 & 35 & 3 & 15 & 21 & 26,25 \\
\hline $52-62$ & 6 & 30 & 2 & 10 & 5 & 25 & 6 & 30 & 19 & 23,75 \\
\hline $63-73$ & 3 & 15 & 8 & 40 & 2 & 10 & 7 & 35 & 20 & 25 \\
\hline Outras & 1 & 5 & 4 & 20 & 1 & 5 & 1 & 5 & 7 & 8,75 \\
\hline \multicolumn{11}{|l|}{ Escolaridade } \\
\hline Sem alfabetização & 5 & 25 & - & - & - & - & 3 & 15 & 8 & 10 \\
\hline Ensino fundamental & 9 & 45 & 4 & 20 & 12 & 60 & 10 & 50 & 35 & 43,75 \\
\hline Ensino médio & 5 & 25 & 10 & 50 & 8 & 40 & 5 & 25 & 28 & 35 \\
\hline Graduação & 1 & 5 & 6 & 30 & - & - & 2 & 10 & 9 & 11,25 \\
\hline Pós-Graduação & - & - & - & - & - & - & - & - & - & - \\
\hline \multicolumn{11}{|l|}{ Renda familiar } \\
\hline$<$ que $1 \mathrm{SM}^{*}$ & 2 & 10 & - & - & 4 & 20 & 3 & 15 & 9 & 11,25 \\
\hline $1-3 \mathrm{SM}$ & 14 & 70 & 10 & 50 & 14 & 70 & 13 & 65 & 51 & 63,75 \\
\hline 4-6 SM & 2 & 10 & 10 & 50 & 2 & 10 & 3 & 15 & 17 & 21,25 \\
\hline 7-9 SM & 1 & 5 & - & - & - & - & - & - & 1 & 1,25 \\
\hline Nenhum & 1 & 5 & - & - & - & - & 1 & 5 & 2 & 2,50 \\
\hline \multicolumn{11}{|l|}{ Convênio } \\
\hline Sim & 4 & 20 & 19 & 95 & 1 & 5 & 3 & 15 & 27 & 33,75 \\
\hline Não & 16 & 80 & 1 & 5 & 19 & 95 & 17 & 85 & 53 & 66,25 \\
\hline
\end{tabular}

Legenda: *SM = salário mínimo (valor vigente na época do estudo: $\mathrm{R} \$ 530,00)$. 
Segundo estudo realizado na cidade de Barcelona, com 4.171 indivíduos de ambos os sexos com idade superior a 14 anos, os indivíduos com nível de escolaridade mais baixo (ensino primário incompleto e completo) apresentaram percepção de saúde regular, má ou muito má (8). Segundo a Pesquisa Nacional por Amostra de Domicílio (PNAD) de 2003, de modo geral, na população brasileira quanto maior a idade e menor a escolaridade maior a prevalência de doenças crônicas (9).

Moreira et al. (6), a fim de avaliar a satisfação pelos serviços de fisioterapia prestados, observaram que a idade média dos pacientes era de 48 anos - dos quais $60 \%$ eram do sexo feminino -, que a escolaridade da amostra era em sua maioria do nível fundamental $(64,5 \%)$, que nos níveis médio e superior de estudo havia $29 \%$ e $6,5 \%$ de pacientes, respectivamente, e que, quanto à renda, $83 \%$ dos indivíduos tinham renda familiar entre um e três salários mínimos.

De acordo com Domínguez-Berjón et al. (8), os estudos socioeconômicos de uma população se fazem importantes para intervenções práticas e fáceis, direcionadas para os desejos, anseios e necessidades da população. 0 conhecimento do perfil do paciente que frequenta as clínicas de fisioterapia permite aos novos investidores e empresários criar soluções rentáveis, adaptadas à realidade da população e que supram as necessidades desta, satisfazendo assim os clientes. Ainda, segundo o mesmo autor, estudos têm mostrado que o perfil socioeconômico de uma população exerce influência na saúde dessa população de forma independente das características individuais (8).

No que se refere à solicitação do tratamento, $52,5 \%$ dos pacientes foram encaminhados por um médico particular e $25 \%$ dos usuários foram submetidos ao tratamento com encaminhamento de SUS externo, ou seja, de outra instituição de saúde. Quanto à facilidade de acesso ao serviço, 78,75\% dos usuários classificam como de fácil acesso, o que é parcialmente explicado pelos $55 \%$ dos entrevistados que se deslocam em veículo próprio ou alugado e pelos $26 \%$ que se deslocam até a clínica caminhando, relatando ela é próxima de suas residências. Em relação à dificuldade com o atendimento, $98 \%$ dos pacientes informam que não apresentaram qualquer dificuldade e que o motivo de escolha do serviço, com $65 \%$, foi a indicação de terceiros ou a proximidade de suas casas, com $31 \%$, reportando à facilidade de acesso (Quadro 2).
Neri (2) mostra a relação direta entre a renda mensal em número de salários mínimos e a posse de plano de saúde. 0 mesmo autor mostra ainda maior prevalência de doenças da coluna, artrites e doenças renais crônicas em indivíduos com menor renda, e maior prevalência de câncer, diabetes e doenças do coração nas populações com maior renda. Ainda, segundo Neri (2), as chances de procurar serviços de saúde aumentam à medida que os indivíduos ganham anos de idade e não acumulam anos de estudo.

Conforme a pesquisa desenvolvida em 2003 pelo Ministério da Saúde junto ao Conselho Nacional de Secretários da Saúde, mais de $90 \%$ da sociedade brasileira utiliza de alguma forma o SUS, sendo um dado satisfatório para o reconhecimento da baixa qualidade dos serviços oferecidos pela rede pública de saúde em relação a equipamentos e serviços profissionais, carência de participação da população na formulação e gestão das políticas de saúde e a falta de mecanismos de acompanhamento, controle e avaliação dos serviços. Nesse contexto, avaliar a satisfação com a assistência torna-se primordial para reconhecer de fato a realidade dos serviços públicos de saúde e promover as alterações necessárias para que se possa alcançar um programa de saúde de qualidade (10).

Acesso e acolhimento são elementos essenciais do atendimento para que se possa incidir efetivamente sobre o estado de saúde do indivíduo e da coletividade (11). A forma de deslocamento utilizada pelo usuário é fator que facilita ou dificulta o seu acesso ao serviço de saúde. A proximidade do serviço de saúde não é o único - tampouco o mais importante - determinante para a escolha do serviço de saúde, visto que nem sempre a proximidade é garantia de atendimento (1). Quando o serviço de saúde é próximo à residência do usuário e a qualidade está garantida, conforme a indicação de familiares, amigos e/ou vizinhos, a opção recai sobre esse serviço (11), o que é evidenciado pelos nossos resultados, que mostram equidade nas opções de indicação por outros, e pela proximidade geográfica do serviço de saúde.

Santos (12) diz que a condição socioeconômica é fator determinante para a escolha do serviço público ou do serviço privado, admitindo que o cuidado médico é semelhante em ambos, variando a rapidez e a facilidade de acesso a alguns procedimentos.

Concernente ao nível de satisfação sobre o tempo de espera para o início da consulta, 93,7\% dos usuários mostraram-se satisfeitos e 90\%, quando 
Quadro 2 - Acessibilidade dos pacientes às clínicas de fisioterapia no município de Campo Maior, PI (2010)

\begin{tabular}{|c|c|c|c|c|c|c|c|c|c|c|}
\hline \multirow[b]{2}{*}{ Dados avaliados } & \multicolumn{2}{|c|}{ Particular } & \multicolumn{2}{|c|}{ Filantrópica } & \multicolumn{2}{|c|}{ Estadual } & \multicolumn{2}{|c|}{ Municipal } & \multicolumn{2}{|c|}{ Total } \\
\hline & $\mathrm{n}=\mathbf{2 0}$ & $\%$ & $\mathrm{n}=\mathbf{2 0}$ & $\%$ & $\mathrm{n}=\mathbf{2 0}$ & $\%$ & $\mathrm{n}=\mathbf{2 0}$ & $\%$ & $\mathrm{n}=80$ & $\%$ \\
\hline \multicolumn{11}{|l|}{ Solicitação } \\
\hline Espontânea & 1 & 5 & 1 & 5 & 1 & 5 & 1 & 5 & 4 & 5 \\
\hline SUS externo & 5 & 25 & - & - & 10 & 50 & 5 & 25 & 20 & 25 \\
\hline SUS interno & - & - & - & - & 5 & 25 & 7 & 35 & 12 & 15 \\
\hline Convênio & - & - & 2 & 10 & - & - & - & - & 2 & 2,50 \\
\hline Particular & 14 & 70 & 17 & 85 & 4 & 20 & 7 & 35 & 42 & 52,50 \\
\hline \multicolumn{11}{|l|}{ Acesso } \\
\hline Muito fácil & 2 & 10 & 6 & 30 & 2 & 10 & - & - & 10 & 12,50 \\
\hline Fácil & 16 & 80 & 14 & 70 & 18 & 90 & 15 & 75 & 63 & 78,75 \\
\hline Difícil & 2 & 10 & - & - & - & - & 5 & 25 & 7 & 9 \\
\hline \multicolumn{11}{|l|}{ Transporte } \\
\hline Próprio ou alugado & 7 & 35 & 17 & 85 & 13 & 65 & 7 & 35 & 44 & 55 \\
\hline Caminhando & 8 & 40 & 2 & 10 & 3 & 15 & 8 & 40 & 21 & 26 \\
\hline Ônibus & 1 & 5 & 1 & 5 & 2 & 10 & 4 & 20 & 8 & 10,00 \\
\hline Bicicleta & 4 & 20 & - & - & 1 & 5 & 1 & 5 & 6 & 8 \\
\hline Carona & - & - & - & - & 1 & 5 & - & - & 1 & 1,25 \\
\hline \multicolumn{11}{|l|}{ Dificuldade } \\
\hline Sim & - & - & - & - & - & - & 1 & 5 & 1 & 1,25 \\
\hline Não & 20 & 100 & 20 & 100 & 20 & 100 & 19 & 95 & 79 & 98,75 \\
\hline \multicolumn{11}{|l|}{ Motivo de escolha } \\
\hline Indicação & 10 & 50 & 15 & 75 & 14 & 70 & 13 & 65 & 52 & 65,0 \\
\hline Não sabe & - & - & - & - & - & - & - & - & - & - \\
\hline Próximo à residência & 10 & 50 & 5 & 25 & 6 & 30 & 4 & 20 & 25 & 31,25 \\
\hline Outros & 0 & - & - & - & - & - & 3 & 15 & 3 & 3,75 \\
\hline
\end{tabular}

perguntados sobre a satisfação pelo tempo de espera para o atendimento, não relataram queixas. Considerando a disponibilidade de aparelhos, $67,5 \%$ dos pacientes afirmam que em momento algum existiu tempo de espera e $26 \%$ contam que às vezes esse tempo de espera acontece, mas é normal. Quanto ao esclarecimento sobre o atendimento a ser prestado, à utilização de técnicas e ao aparato técnico, 88,7\% dos usuários alegam que o profissional fornece informações sobre o serviço. Dentre os entrevistados, $62,5 \%$ expressam ótima relação com o fisioterapeuta e 37,5\% mostram um bom relacionamento com os demais profissionais (Quadro 3).

Em todo o processo diagnóstico e terapêutico, a familiaridade, a confiança e a colaboração estão altamente implicadas no resultado da arte médica (10). A desumanização dos serviços de saúde como um todo tem sido centro de muitas discussões, especialmente quanto à percepção do paciente $\mathrm{e}$ as possíveis implicações sobre sua saúde e estado psicológico (4). A doença é interpretada, pela concepção biomédica, como um desvio de variáveis biológicas em relação à norma. Esse modelo, fundamentado em uma perspectiva mecanicista, considera os fenômenos complexos como sendo constituídos por princípios simples, isto é, relação de causa e efeito, distinção cartesiana entre mente e corpo, análise do corpo como máquina, minimizando os aspectos sociais, psicológicos e comportamentais (10). 
Quadro 3 - Avaliação quanto à relação profissional/paciente dos indivíduos entrevistados em quatro clínicas no município de Campo Maior, PI (2010)

\begin{tabular}{|c|c|c|c|c|c|c|c|c|c|c|}
\hline \multirow[b]{2}{*}{ Dados avaliados } & \multicolumn{2}{|c|}{ Particular } & \multicolumn{2}{|c|}{ Filantrópica } & \multicolumn{2}{|c|}{ Estadual } & \multicolumn{2}{|c|}{ Municipal } & \multicolumn{2}{|c|}{ Total } \\
\hline & $\mathrm{n}=\mathbf{2 0}$ & $\%$ & $\mathrm{n}=\mathbf{2 0}$ & $\%$ & $\mathrm{n}=\mathbf{2 0}$ & $\%$ & $\mathrm{n}=\mathbf{2 0}$ & $\%$ & $\mathrm{n}=80$ & $\%$ \\
\hline \multicolumn{11}{|c|}{ Satisfação espera para consulta } \\
\hline Muito satisfeito & - & - & 1 & 5 & - & - & - & - & 1 & 1,25 \\
\hline Satisfeito & 20 & 100 & 19 & 95 & 18 & 90 & 18 & 90 & 75 & 93,75 \\
\hline Mais ou menos & - & - & - & - & 1 & 5 & 1 & 5 & 2 & 2,50 \\
\hline Insatisfeito & - & - & - & - & 1 & 5 & 1 & 5 & 2 & 2,50 \\
\hline Muito insatisfeito & - & - & - & - & - & - & - & - & - & - \\
\hline \multicolumn{11}{|c|}{ Satisfação espera para atendimento } \\
\hline Muito satisfeito & - & - & 1 & 5 & - & - & - & - & 1 & 1,25 \\
\hline Satisfeito & 20 & 100 & 19 & 95 & 19 & 95 & 14 & 70 & 72 & 90 \\
\hline Mais ou menos & - & - & - & - & 1 & 5 & 5 & 25 & 6 & 7,50 \\
\hline Insatisfeito & - & - & - & - & - & - & 1 & 5 & 1 & 1,25 \\
\hline Muito insatisfeito & - & - & - & - & - & - & - & - & - & - \\
\hline \multicolumn{11}{|c|}{ Espera por falta de aparelho } \\
\hline Sim & - & - & - & - & 1 & 5 & 2 & 10 & 3 & 2,75 \\
\hline Não & 17 & 85 & 19 & 95 & 9 & 45 & 9 & 45 & 54 & 67,50 \\
\hline Às vezes & 3 & 15 & 1 & 5 & 8 & 40 & 9 & 45 & 21 & 26,25 \\
\hline Não utiliza & - & - & - & - & 2 & 10 & - & - & 2 & 2,50 \\
\hline \multicolumn{11}{|l|}{ Esclarecimento } \\
\hline Sim & 19 & 95 & 20 & 100 & 19 & 95 & 13 & 65 & 71 & 88,75 \\
\hline Não & 1 & 5 & - & - & 1 & 5 & 7 & 35 & 9 & 11,25 \\
\hline \multicolumn{11}{|c|}{ Relação com fisioterapeuta } \\
\hline Ótimo & 10 & 50 & 18 & 90 & 12 & 60 & 10 & 50 & 50 & 62,50 \\
\hline Bom & 10 & 50 & 2 & 10 & 8 & 40 & 10 & 50 & 30 & 37,50 \\
\hline Regular & - & - & - & - & - & - & - & - & - & - \\
\hline Ruim & - & - & - & - & - & - & - & - & - & - \\
\hline Péssimo & - & - & - & - & - & - & - & - & - & - \\
\hline
\end{tabular}

Segundo Sampaio (13), a fisioterapia tem crescido de forma espantosa e desorganizada nos últimos anos, o que pode enviesar o horizonte humano do tratamento fisioterapêutico. Além disso, a fisioterapia historicamente provém da medicina, herdando um pensamento cartesiano e mediocêntrico, pensamento esse enriquecido e fortalecido pelas universidades, que muitas vezes não se mostram preocupadas com a reinserção do paciente na sociedade (14).

Considerando a ambiência das clínicas, 53,7\% dos indivíduos julgam a limpeza como ótima e 40\% classificaram como boa. Quanto ao conforto, $50 \%$ dos pacientes optaram por ótimo e $40 \%$ afirmam ter um bom conforto. No que se refere às instalações, 47,5\% dos entrevistados escolheram a opção ótima e $40 \%$ relataram ser boas e adequadas para o ambiente analisado; $90 \%$ dos pacientes asseguraram ter sua privacidade respeitada durante o atendimento e $100 \%$ relatam que as quatro clínicas avaliadas atendem às suas necessidades de tratamento (Quadro 4).

A satisfação do paciente com a assistência recebida é influenciada por características sociodemográficas dos usuários, em especial no tocante ao sexo 
Quadro 4 - Avaliação da ambiência de quatro clínicas no município de Campo Maior, PI (2010)

\begin{tabular}{|c|c|c|c|c|c|c|c|c|c|c|}
\hline \multirow[b]{2}{*}{ Dados avaliados } & \multicolumn{2}{|c|}{ Particular } & \multicolumn{2}{|c|}{ Filantrópica } & \multicolumn{2}{|c|}{ Estadual } & \multicolumn{2}{|c|}{ Municipal } & \multicolumn{2}{|c|}{ Total } \\
\hline & $\mathrm{n}=\mathbf{2 0}$ & $\%$ & $\mathrm{n}=\mathbf{2 0}$ & $\%$ & $\mathrm{n}=\mathbf{2 0}$ & $\%$ & $\mathrm{n}=\mathbf{2 0}$ & $\%$ & $\mathrm{n}=80$ & $\%$ \\
\hline \multicolumn{11}{|l|}{ Limpeza } \\
\hline Ótimo & 11 & 55 & 16 & 80 & 12 & 60 & 4 & 20 & 43 & 53,75 \\
\hline Bom & 6 & 30 & 4 & 20 & 8 & 40 & 14 & 70 & 32 & 40 \\
\hline Regular & 3 & 15 & 0 & - & - & - & 1 & 5 & 4 & 5 \\
\hline Ruim & - & - & - & - & - & - & 1 & 5 & 1 & 1,25 \\
\hline Péssimo & - & - & - & - & - & - & - & - & - & - \\
\hline \multicolumn{11}{|l|}{ Conforto } \\
\hline Ótimo & 12 & 60 & 16 & 80 & 8 & 40 & 4 & 20 & 40 & 50 \\
\hline Bom & 5 & 25 & 4 & 20 & 12 & 60 & 11 & 55 & 32 & 40 \\
\hline Regular & 3 & 15 & - & - & - & - & 5 & 25 & 8 & 10 \\
\hline Ruim & - & 0 & - & - & - & - & - & - & - & - \\
\hline Péssimo & - & - & - & - & - & - & - & - & - & - \\
\hline \multicolumn{11}{|l|}{ Instalações } \\
\hline Ótimo & 12 & 60 & 13 & 65 & 8 & 40 & 5 & 25 & 38 & 47,50 \\
\hline Bom & 7 & 35 & 7 & 35 & 9 & 45 & 9 & 45 & 32 & 40 \\
\hline Regular & 1 & 5 & - & - & 2 & 10 & 6 & 30 & 9 & 11,25 \\
\hline Ruim & - & - & - & - & 1 & 5 & - & - & 1 & 1,25 \\
\hline Péssimo & - & - & - & - & - & - & - & - & - & - \\
\hline \multicolumn{11}{|l|}{ Privacidade } \\
\hline Sim & 20 & 100 & 20 & 100 & 19 & 95 & 13 & 65 & 72 & 90 \\
\hline Não & 0 & - & - & - & 1 & 5 & 7 & 35 & 8 & 10 \\
\hline \multicolumn{11}{|l|}{ Necessidade } \\
\hline Sim & 20 & 100 & 20 & 100 & 20 & 100 & 20 & 100 & 80 & 100 \\
\hline
\end{tabular}

do paciente, à renda familiar e ao nível educacional (6). A satisfação desses indivíduos é essencial e importante para a obtenção da qualidade da atenção, pois irá revelar ao profissional informações a respeito dos valores e expectativas dos pacientes, que esperam do serviço a qualidade desejada (15). A satisfação de cada usuário sobre os serviços de saúde é uma forma de avaliação necessária para que os profissionais compreendam suas necessidades quanto aos serviços prestados, considerando suas subjetividades e sua percepção sobre o processo de trabalho (11).

Em sentido mais amplo, essas pesquisas permitem verificar o modo como os direitos individuais e de cidadania são observados no acesso e na utilização dos serviços e sistemas de saúde (13).

Vários autores concordam que os usuários expressam alto grau de satisfação com a dimensão relacional em todos os seus atributos: respeito, consideração, escuta, compreensão, acolhida e gentileza por parte dos profissionais da equipe (6). Esse fato evidencia-se pela quantidade de indivíduos que relataram respeito à privacidade, ótimo relacionamento com os profissionais, além de conforto, boas instalações, limpeza e a satisfação de suas necessidades.

Ressalta-se que neste estudo observamos que, nas clínicas municipais e estaduais, o grau de satis- 
fação dos usuários foi um pouco menor em razão, principalmente, do maior tempo de espera, tanto pelo atendimento quanto por aparelho, para o tratamento. Inúmeras vezes o serviço público de saúde é sobrecarregado por causa da precarização tanto de aparelhos quanto da mão de obra.

Segundo Mendonça (4), a dimensão da interação paciente/terapeuta encontra-se fortemente correlacionada com a satisfação do paciente e também indica que a comunicação entre o profissional e seu paciente pode ser o principal elo que permeia os diversos aspectos que compõem o processo da assistência fisioterapêutica. Keith (3) afirma que a percepção da qualidade do atendimento parte da interação interpessoal terapeuta/paciente passando pelas atribuições técnicas e permeadas pela comunicação durante as consultas e atendimentos.

Quanto aos dados referentes à ambiência, percebe-se menor satisfação, no que condiz à limpeza, ao conforto, à privacidade e às instalações, dos indivíduos atendidos na clínica municipal se olharmos as outras clínicas, no entanto, independente das queixas, as necessidades foram supridas.

\section{Considerações finais}

Com base na pesquisa realizada, observou-se grande satisfação dos usuários dos serviços de fisioterapia do município de Campo Maior, relatando-se, entretanto, pequena insatisfação dos usuários dos serviços estaduais e municipais por conta da espera por atendimento, por aparelho, conforto, estrutura e limpeza do ambiente. Contudo, todos os pacientes entrevistados, de todas as clínicas, relataram a satisfação de suas necessidades.

Quanto ao perfil socioeconômico, a maioria dos entrevistados era do sexo feminino (55\%) - confirmando algo já demonstrado pela literatura mundial: que o público feminino procura mais pelos serviços de saúde -, a maior parte tinha entre 41 e 50 anos, recebia de um a três salários mínimos, possuía o ensino fundamental e não tinha plano de saúde.

Outros estudos se fazem necessários em populações maiores, correlacionando o grau de satisfação aos dados socioeconômicos, a fim de confirmar as tendências observadas neste estudo e de avaliar quais são os fatores determinantes para a satisfação dos usuários de fisioterapia.

\section{Referências}

1. Gouveia GC, de Souza WV, Luna CF, de Souza-Júnior PRB, Szwarcwald CL. Satisfação dos usuários do sistema de saúde brasileiro: fatores associados e diferenças regionais. Rev Bras Epidemiol. 2009;12(3): 281-96.

2. Neri M, Soares W. Desigualdade social e saúde no Brasil. Cad Saúde Pública. 2002;18:77-87.

3. Keith RA. Patient satisfaction and rehabilitation services. Arch Phys Med Rehabil. 1998;79(9):1122-8.

4. Mendonça KMPP, Guerra RO. Desenvolvimento e validação de um instrumento de medida da satisfação do paciente com a fisioterapia. Rev Bras Fisioter. 2007; 11(5):369-76.

5. Goldstein MS, Elliot SD, Guccione AA. The development of an instrument to measure satisfaction with physical therapy. Phys Ther. 2000:80(9):853-63.

6. Moreira CF, Borba JAM, Mendonça KMPP. Instrumento para aferir a satisfação do paciente com a assistência fisioterapêutica na rede pública de saúde. Fisioterapia e Pesquisa. 2007;14(3):37-43.

7. Machado NP, Nogueira LT. Avaliação da satisfação dos usuários de serviços de fisioterapia. Rev Bras Fisioter. 2008;12(5):401-8.

8. Domínguez-Berjón MF, Borrell C, Pastor V. Indicadores socioeconómicos de área pequeña en el estudio de las desigualdades en salud. Gac Sanit. 2004; 18(2):92-100.

9. Barros MBA, César CLG, Carandina L, Torre GD. Desigualdades sociais na prevalência de doenças crônicas no Brasil, PNAD-2003. Ciênc Saúde Colet. 2006; 11(4):911-926.

10. Caprara A, Franco ALS. A relação paciente-médico: para uma humanização da prática médica. Cad Saúde Pública. 1999;15(3):647-54.

11. de Paiva SMA, Gomes ELR. Assistência hospitalar: avaliação da satisfação dos usuários durante seu período de internação. Rev Latino-Am Enfermagem. 2007;15(5):973-9.

12. Santos MP. Avaliação da qualidade dos serviços públicos de atenção à saúde da criança sob a ótica do usuário. Rev Bras Enferm. 1995;48(2):99-106. 
13. Sampaio RF. Promoção de saúde, prevenção de doenças e incapacidade: a experiência da fisioterapia/ UFMG em unidade básica de saúde. Fisioter Mov. 2002;15(1):19-24.

14. Galheigo MS. A transdisciplinaridade enquanto princípio e realidade das ações de saúde. Rev Terap Ocupac. 1999;10(2-3):49-54.
15. Mendes ACG, de Araújo Jr JLCA, Furtado BMAS, Duarte PO, Santiago RF, da Costa TR. Avaliação da satisfação dos usuários com a qualidade do atendimento nas grandes emergências do Recife, Pernambuco, Brasil. Rev Bras Saude Mater Infant. 2009;9:157-165.

Recebido: 21/07/2010 Received: 07/21/2010

Aprovado: $18 / 11 / 2010$

Approved: 11/18/2010 